\title{
Regulation of Lipoprotein Homeostasis by Self-assembling Peptides
}

Victoria Harbour ${ }^{1}$, Candice Casillas $^{2}$, Zain Siddiqui ${ }^{1}$, Biplab Sarkar ${ }^{1}$, Sreya Sanyal ${ }^{1}$, Peter Nguyen ${ }^{1}, \mathrm{Ka}$ Kyung Kim ${ }^{1}$, Abhishek Roy ${ }^{1}$, Patricia Iglesias-Montoro ${ }^{1}$, Saloni Patel ${ }^{1}$, Frank Podlaski², Peter Tolias ${ }^{2}$, William Windsor ${ }^{2}$, Vivek Kumar ${ }^{1,3,4}$ *

${ }^{1}$ Department of Biomedical Engineering, New Jersey Institute of Technology, Newark, NJ, 07102, USA

${ }^{2}$ Department of Chemistry and Chemical Biology, Stevens Institute of Technology, Hoboken, NJ 07030

${ }^{3}$ Department of Chemical and Materials Engineering, New Jersey Institute of Technology, Newark, NJ, 07102, USA

${ }^{4}$ Department of Restorative Dentistry, Rutgers School of Dental Medicine, Newark, NJ, 07103, USA

\section{Supplemental Materials:}

Figure S1. Ultrastructural characterization of peptide constructs.

Figure S2. Comprehensive rheological properties of constructs

Figure S3. Chemical analysis and Binding properties of a control peptide with a nonspecific mimic.

Figure S4. Binding of peptide onto carbonic anhydrous surfaces.

Figure S5. Mass Spectrometry. 


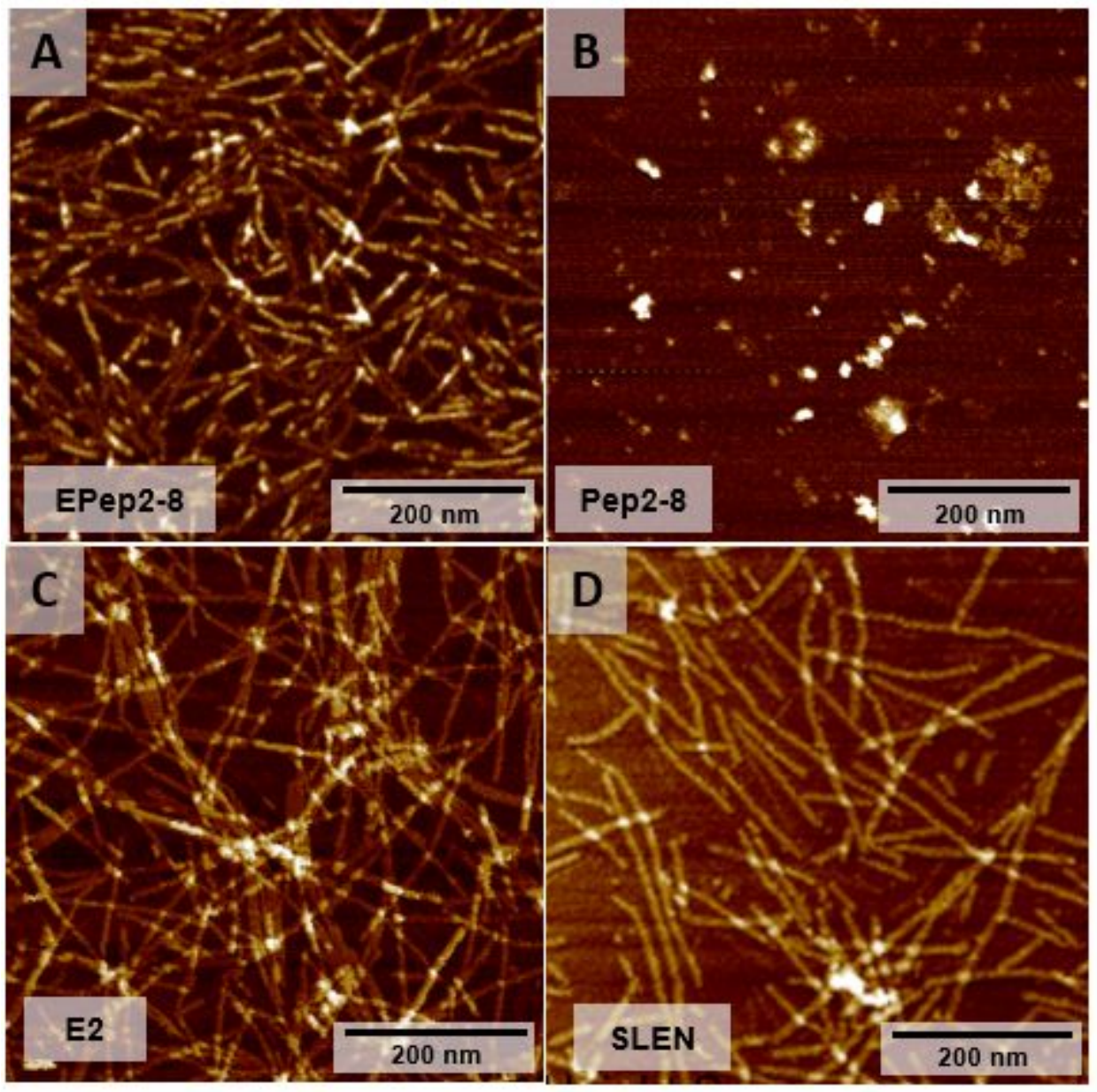

Figure S1. Ultrastructural characterization of peptide constructs. (A) Atomic force microscopy (AFM) of EPep2-8 shows nanofiber formation characteristic of hydrogels within this platform. (B) Pep2-8 lacks a self-assembling domain and does not form a hydrogel, evident from the lack of nanofibrous structures and formation of aggregates. (C) The self assembling domain, E2, also forms characteristic fibers (width $8.7 \mathrm{~nm} \pm 1.2 \mathrm{~nm}$ ), when conjugated to other peptide mimicking domains such as neurogenic ependymin (SLen), fiber width $10.2 \mathrm{~nm} \pm 1.4 \mathrm{~nm}(\mathrm{D})$. 

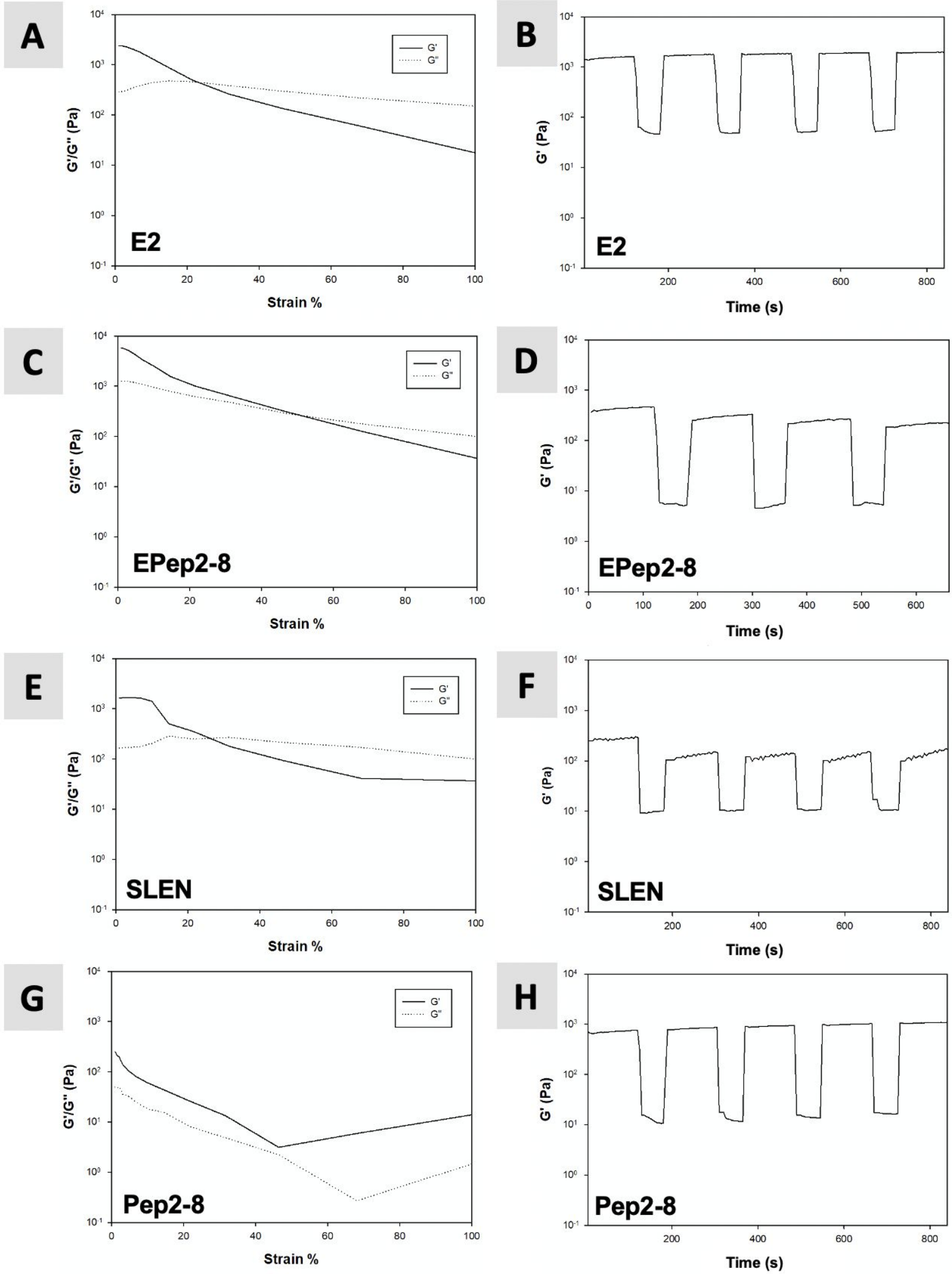

Figure S2. Comprehensive rheological properties of constructs. (A, C, E) At high strain, hydrogels exhibit significant shear thinning demonstrated by inversion of elastic and viscous moduli. At low strain, hydrogel storage moduli (G') exceed loss moduli (G”). (G) Viscous solutions of Pep2-8 do not shear-thin as observed by a lack of $G$ '/G' inversion upon high shear strain. (B, D, F, H) Minimal hysteresis is observed after repeated cyclic loading at low 
and high strain rates. Peptide storage moduli (G') decrease at high strain (100\% for $60 \mathrm{~s})$ and immediately recover following strain removal ( $1 \%$ for $120 \mathrm{~s})$.
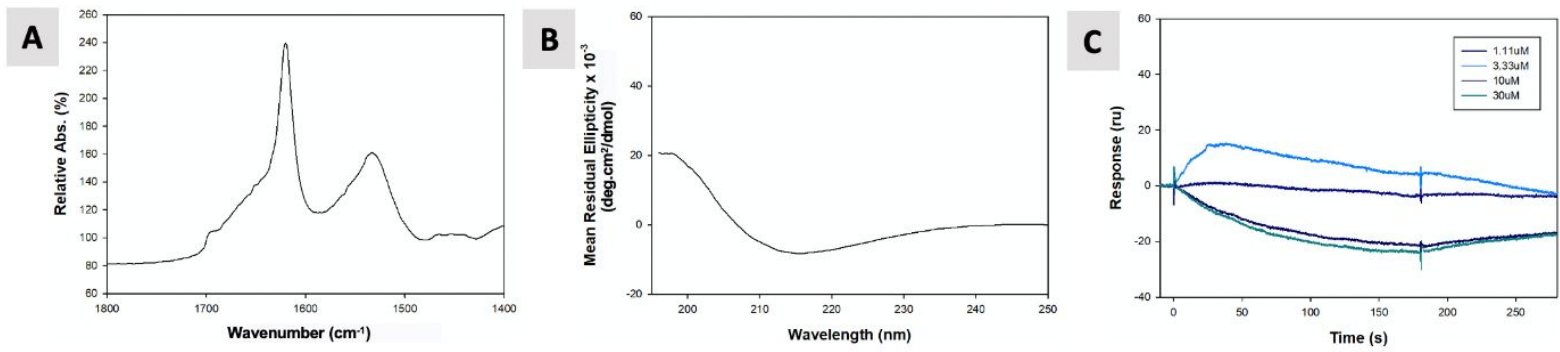

Figure S3. Chemical analysis and Binding properties of a control peptide with a nonspecific mimic. (A) ATR-FTIR of SLen shows a $\square$-sheet-specific amide-1 characteristic peak at $(\sim 1622 \mathrm{~cm}-1)$. SLen is a neurogenic self-assembling peptide evaluated by our group, used here as a control. (B) Circular dichroism of SLen shows characteristic minima at $\sim 215 \mathrm{~nm}$, further indicating nanofibers adopt $\square$-sheet secondary structure. (C) No discernable binding affinity was noted between SLen and PCSK9.
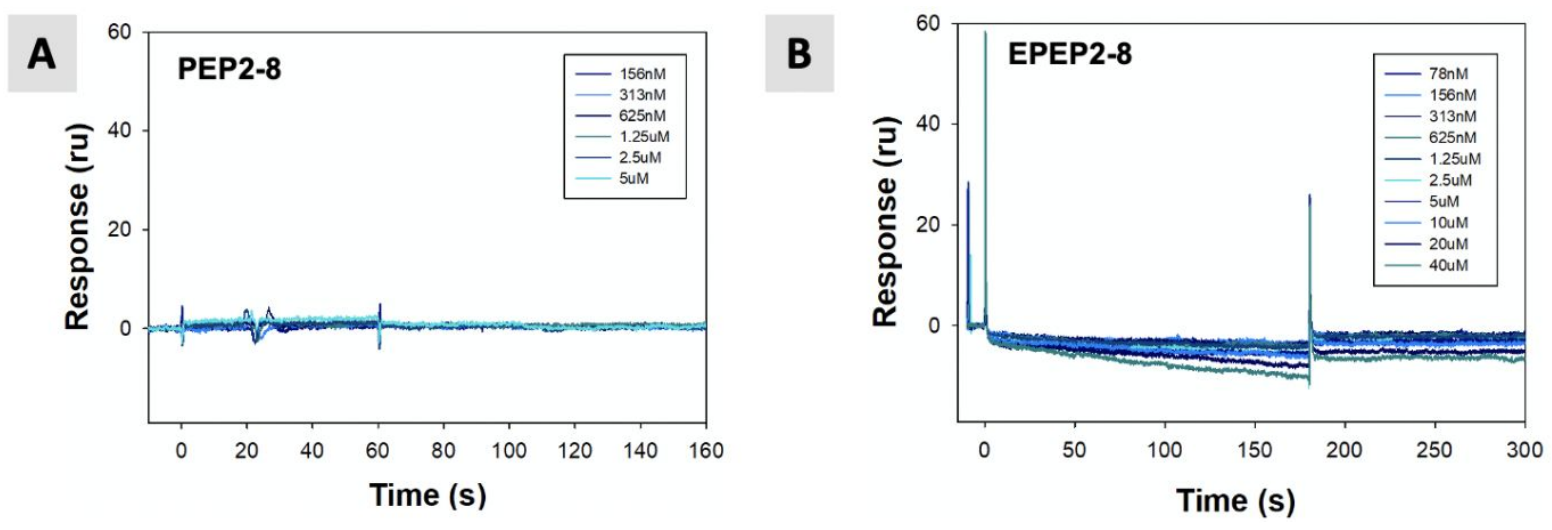

Figure S4. Binding of peptide onto carbonic anhydrous surfaces. (A) Pep2-8 and (B) EPep2-8 do not show appreciable binding to a control CAII surface.
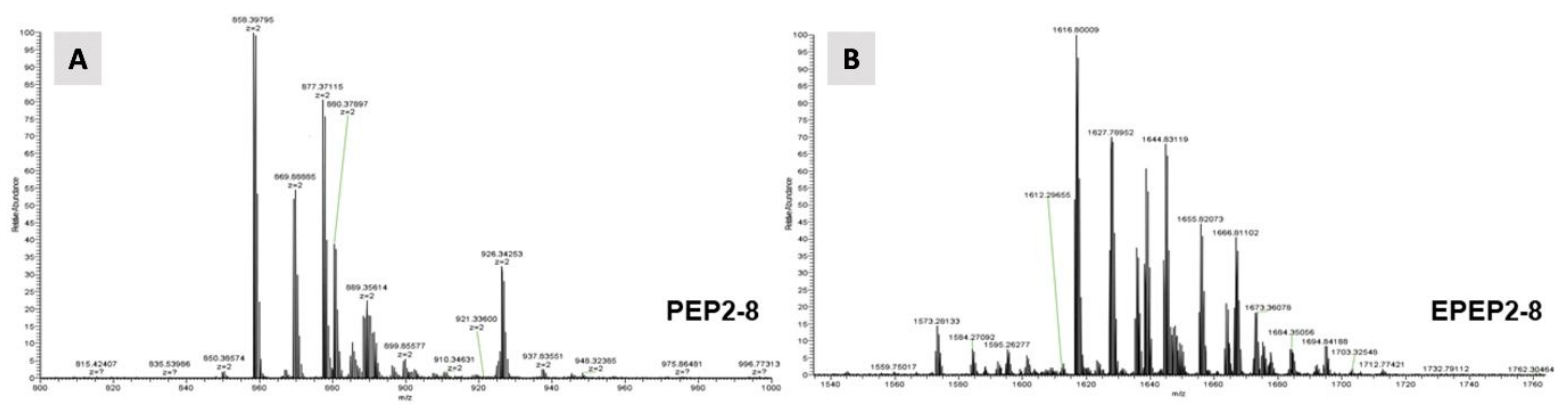

Figure S5. Mass Spectrometry. Solid state synthesis yielded pure samples of (A) Pep2-8 (Mw $1715.85 \mathrm{~g} / \mathrm{mol}$ ) and (B) EPep2-8 (Mw $3232.54 \mathrm{~g} / \mathrm{mol})$. Other peaks indicate interaction with sodium ions ( $\mathrm{Mw} 23 \mathrm{~g} / \mathrm{mol}$ ), which were introduced during titration of peptide solutions to $\mathrm{pH} 7$ with $\mathrm{NaOH}$. 